Europ. Neurol. 1978;17:I-V

Contents, Vol. 17, 1978

Founded 1897 as 'Monatsschrift fur Psychiatric und Neurologie', continued 1957-1967 as 'Psychiatria et Neurologia'

Founders: C. Wernicke and Th. Ziehen

Successors: K. Bonhoeffer (1912-1938), J. Klaesi (1939-1967), E. Grunthal (1953-1967)

Editor-in-Chief:

H.E. Kaeser, Basel

Editorial Board:

L. Barraquer Bordas, Barcelona

J. Droogleever Fortuyn,

Groningen
Associate Editors:

C. Loeb, Genova

U.K. Rinne, Turku

H.-G. Mertens, Wurzburg H. vander Eecken, Ghent
P. Huber, Bern
S. Feldman, Jerusalem
P. Passouant, Montpellier
E. Satoyoshi, Tokyo
A. Kreindler, Bucharest F. Seitelberger, Vienna
H.J. Lehmann, Essen $\quad$ F. Vassella, Bern 
S. Karger $\bullet$ Basel $\bullet \mid$ Mïnchen $\bullet$ Paris $\bullet$ London $\bullet$ New York $\bullet$ Sydney 
S. Karger AG,

4011 Basel (Switzerland), Arnold-Bocklin-Strasse 25

Printed in Switzerland by Thiir AG Offsetdruck,

Pratteln 
All rights reserved.

No part of this publication may be translated into other languages, reproduced or utilized in any form or by any means, electronic or mechanical, including photocopying, recording, microcopying, or by any information storage and retrieval system, without permission in writing from the publisher. 
Contents

No. 1 


\section{No. 2}

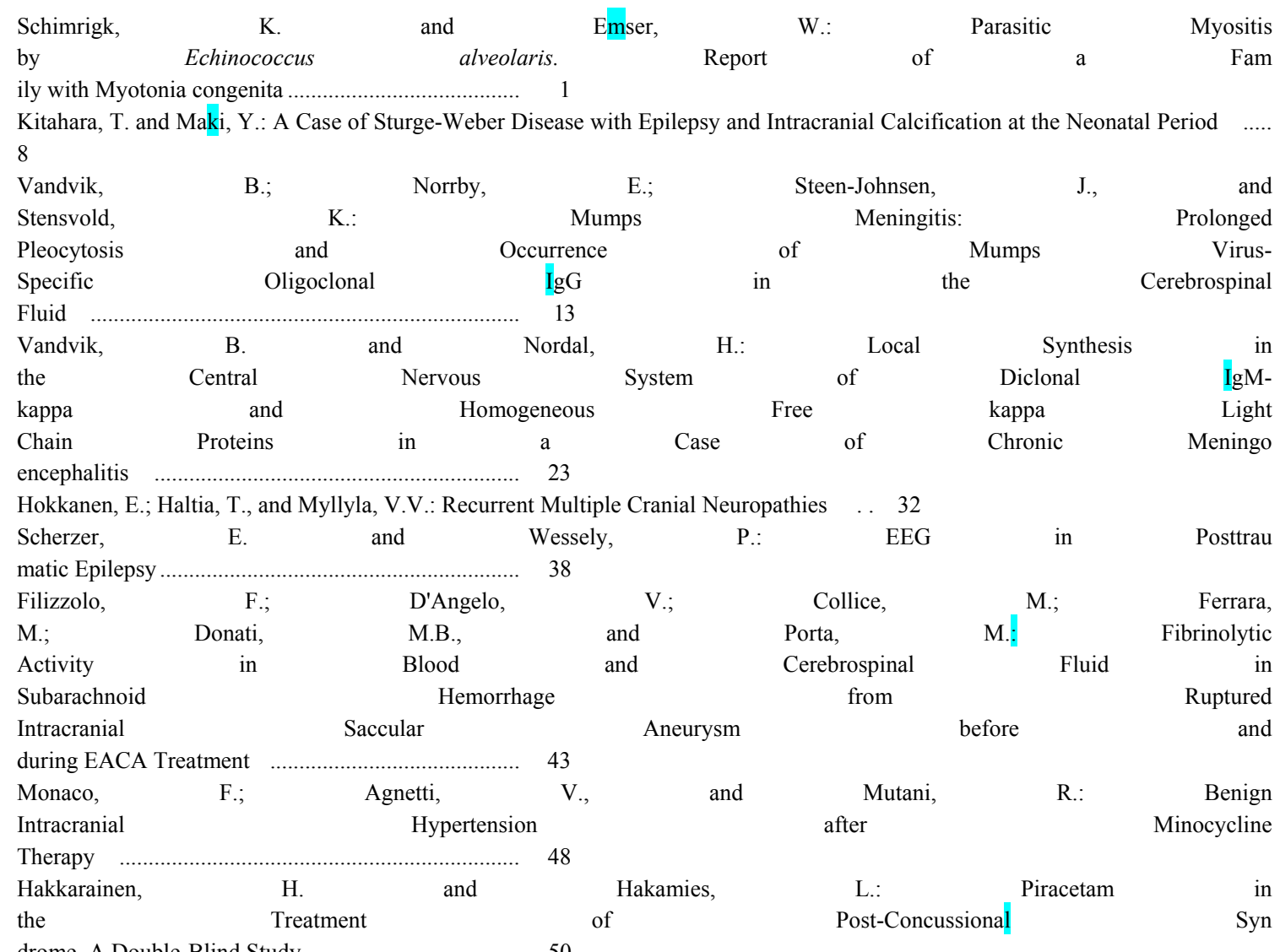

drome. A Double-Blind Study

50

De Zanche, L.; Negrin, P.; Fardin, P., and Carteri, A.: Paralysis of the Deep Branch of the Radial Nerve Due to an Entrapment Neuropathy. 56 


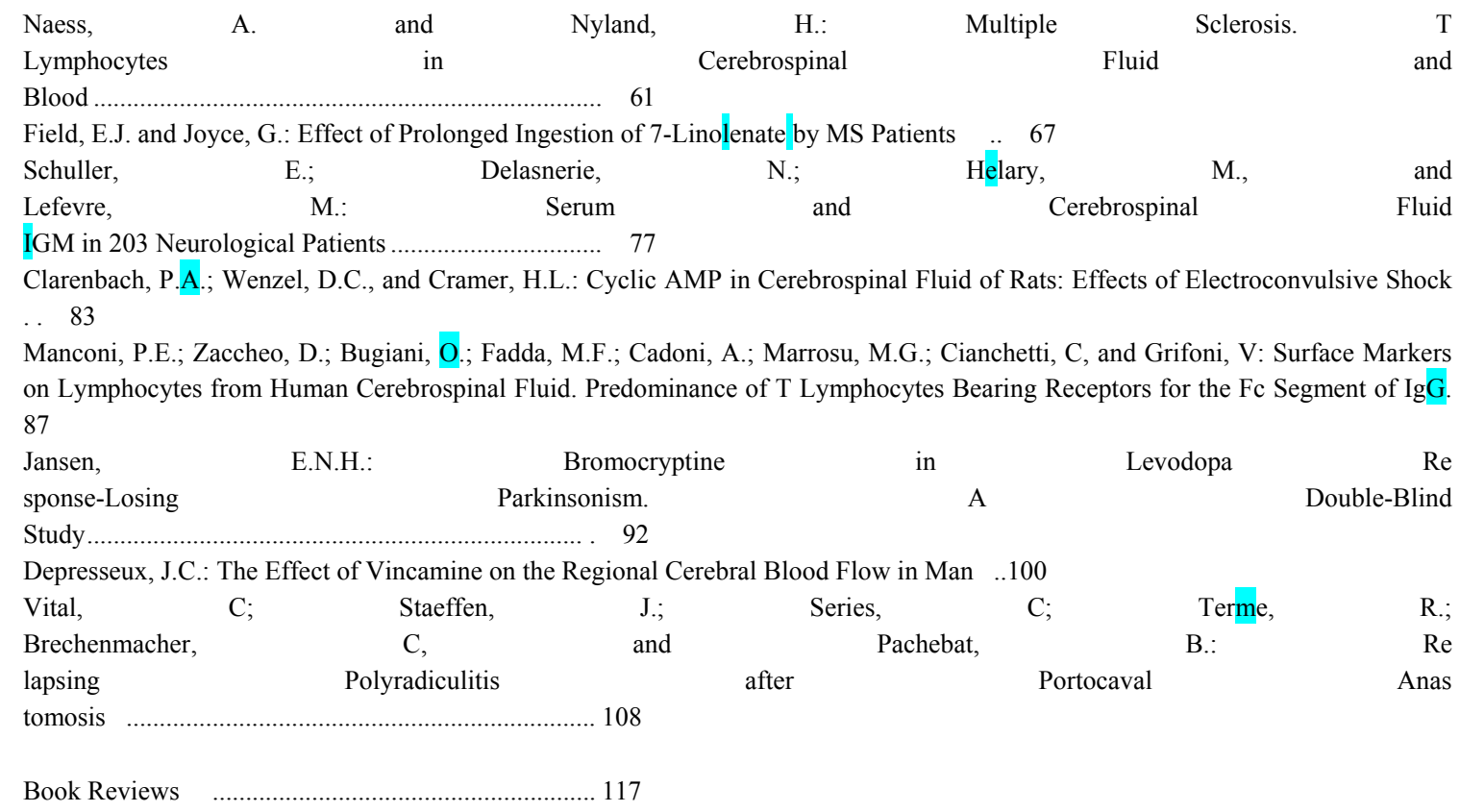

\section{No. 3}

\begin{tabular}{|c|c|c|c|c|c|c|}
\hline $\begin{array}{l}\text { Gall, } \\
\mathrm{H}:\end{array}$ & $\begin{array}{c}\text { M. } \\
\text { Absence }\end{array}$ & $\begin{array}{l}\text { von; } \\
\text { Status }\end{array}$ & $\begin{array}{l}\text { Scollo-Lavizzari, } \\
\text { in the }\end{array}$ & $\begin{array}{l}\text { G., } \\
\text { Adult. }\end{array}$ & $\begin{array}{l}\text { and } \\
\text { New }\end{array}$ & $\begin{array}{l}\text { Becker, } \\
\text { Results }\end{array}$ \\
\hline Including & & Con & & Transverse & & Axial \\
\hline
\end{tabular}


Nohl, M.; Doose, H.; Gross-Selbeck, G., and Jensen, H.-P.: Spinal Myoclonus

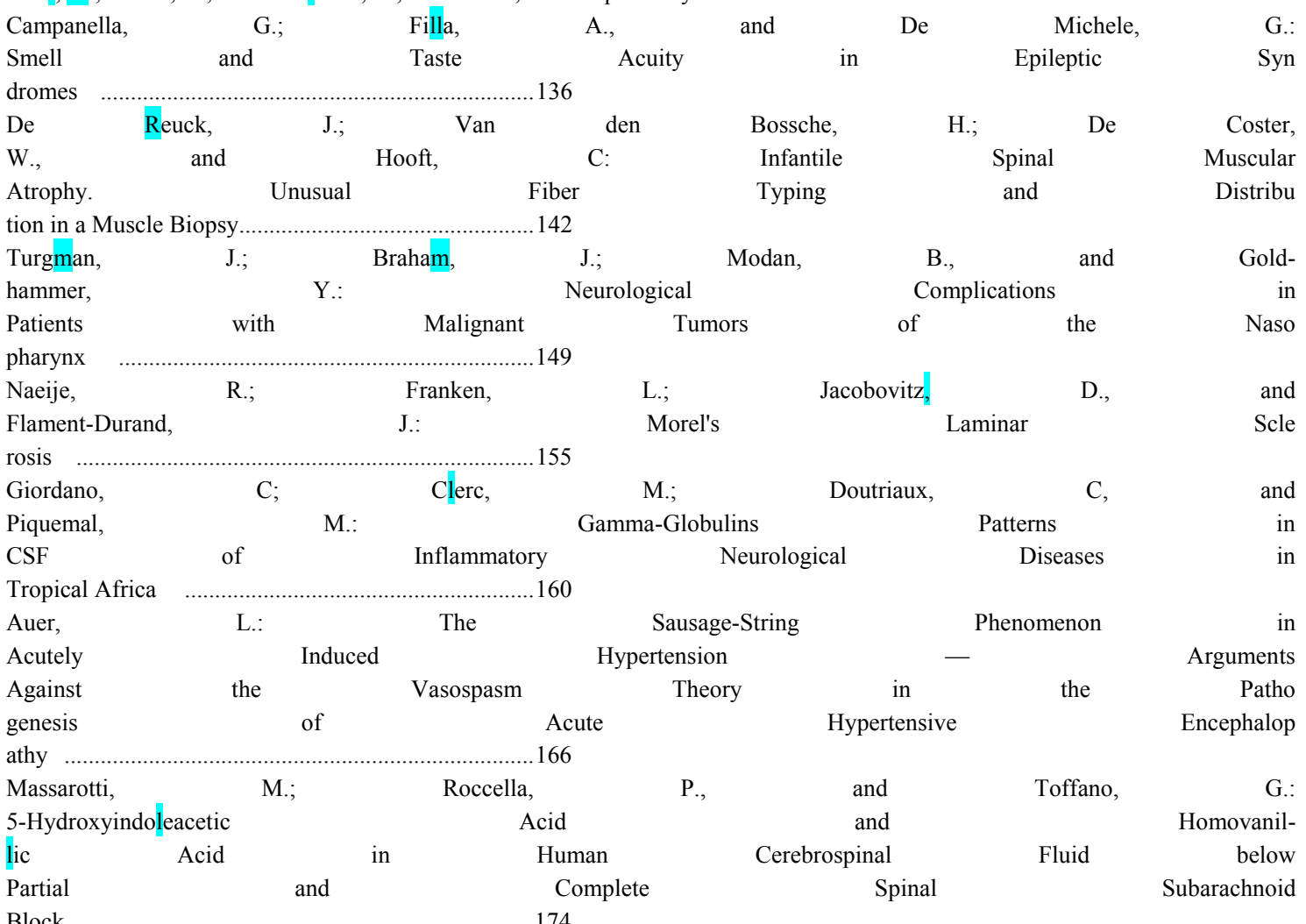

\section{No. 4}

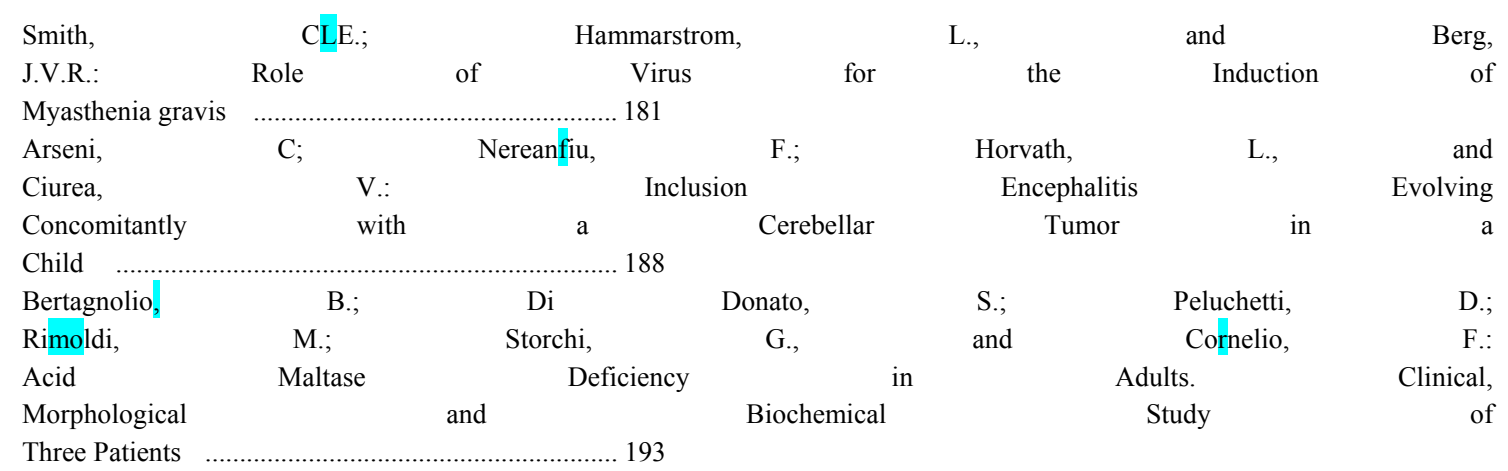


Johansson, B.B.; Linder, L.-E., and Borenstein, P.: Cerebrovascular Permeability in Acute Arterial Hypertension. Effect of Unilateral Ligation of the Common Carotid Artery . . 205

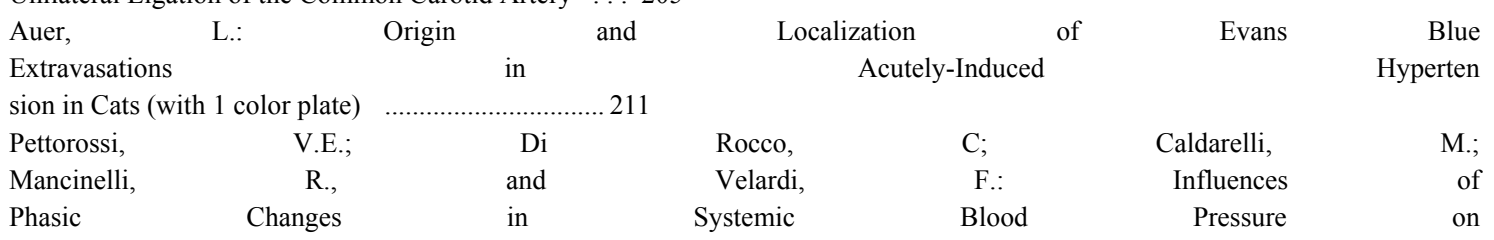

Intracranial Pressure $\quad$................................................. 216

Dereymaeker, A.; Lams-Cauwe, V., and Fobelets, P.: Frontal Dynamic Thermometry. Improvement in Diagnosis of Carotid Stenosis .... 226

\begin{tabular}{|c|c|c|c|c|c|c|}
\hline $\begin{array}{l}\text { Alexianu, } \\
\text { dorescu, } \\
\text { Neurolipidosis. }\end{array}$ & $\begin{array}{l}\text { M.; } \\
\text { D., }\end{array}$ & $\begin{array}{l}\text { Oancea, } \\
\text { and } \\
\text { Ultrastructural }\end{array}$ & $\begin{array}{r}\text { C; } \\
\text { Ciurea, }\end{array}$ & $\begin{array}{l}\text { Petrovici, } \\
\text { V.: } \\
\text { dy }\end{array}$ & $\begin{array}{r}\text { Al.; } \\
\text { Atypical } \\
\text { of }\end{array}$ & $\begin{array}{r}\text { Christo- } \\
\text { Juvenile } \\
\text { a }\end{array}$ \\
\hline
\end{tabular}

Cerebral Biopsy

Book Reviews

239

\section{No. 5}

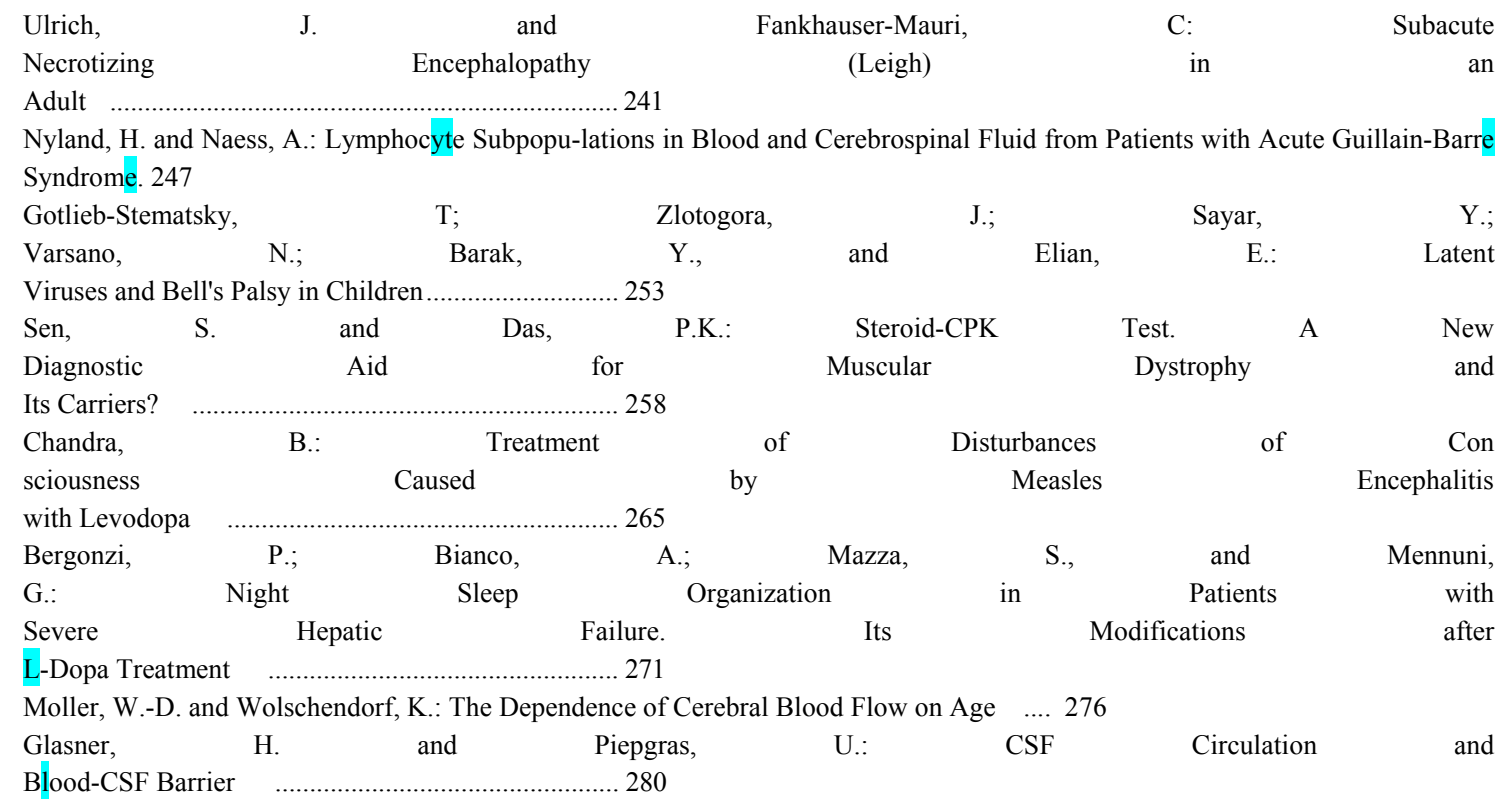




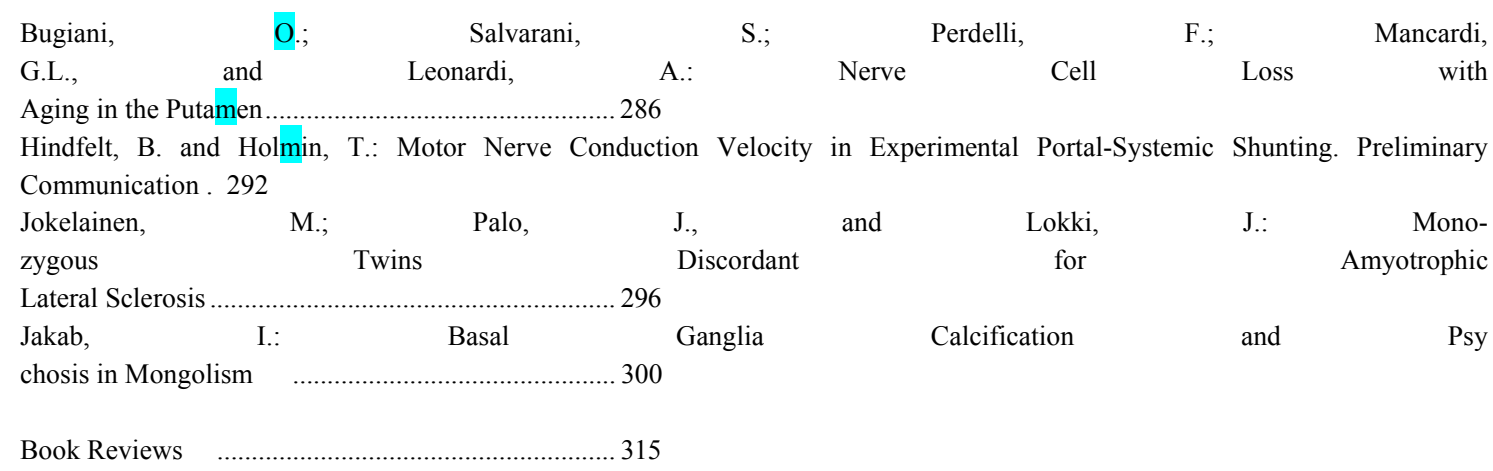

\section{No. 6}

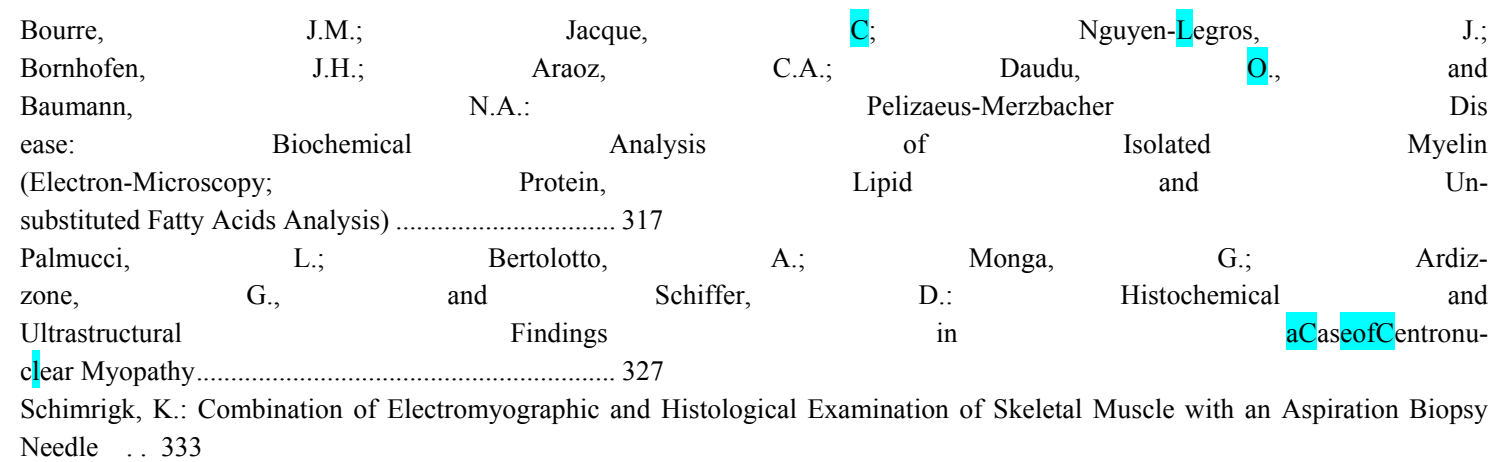




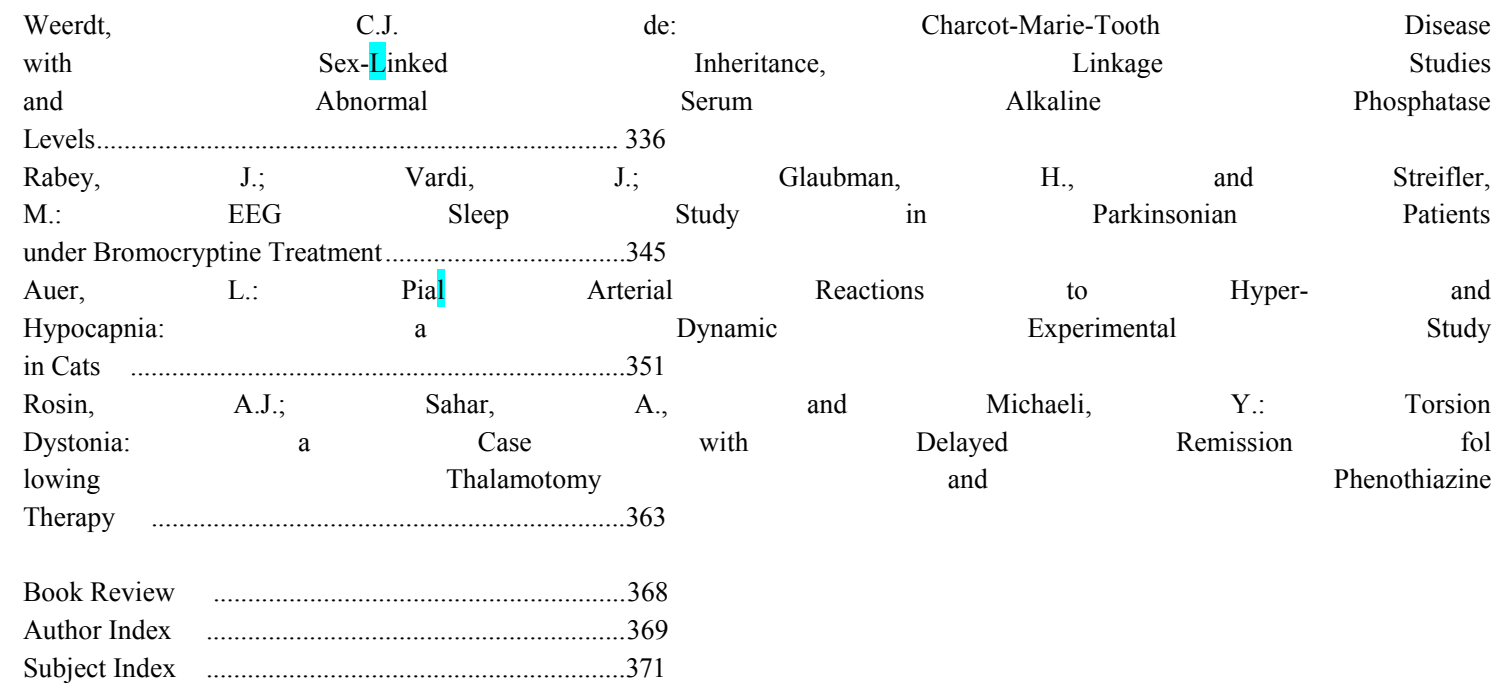

\section{Supplement 1}

Medical Management of Cerebral Ischemia Proceedings of a Symposium on Medical Therapy of Cerebral Ischemia - Recent Advances in Pharmacology and Therapy: (-)Eburnamonine, Milano, December 9-10, 1977 